\title{
1 Targeting glucose metabolism sensitizes pancreatic cancer to MEK inhibition
}

3 Liang Yan', Bo Tu${ }^{1}$, Jun $\mathrm{Yao}^{1}$, Jing Gong ${ }^{2}$, Alessandro Carugo ${ }^{3}$, Christopher A. Bristow ${ }^{3}$,

4 Qiuyun Wang ${ }^{1}$, Cihui Zhu' ${ }^{1}$, Bingbing Dai ${ }^{4}$, Ya'an Kang ${ }^{4}$, Leng $\mathrm{Han}^{2}$, Ningping Feng ${ }^{3}$, Yanqing

$5 \quad$ Jin $^{1}$, Jason Fleming ${ }^{4,5}$, Timothy P. Heffernan ${ }^{3}$, Wantong Yao ${ }^{6}$, Haoqiang Ying ${ }^{1}$

$6{ }^{1}$ Department of Molecular and Cellular Oncology, University of Texas MD Anderson Cancer

7 Center

$8 \quad 2$ Department of Biochemistry and Molecular Biology, UTHealth Medical School

$9 \quad{ }^{3}$ Translational Research to Advance Therapeutics and Innovation in Oncology (TRACTION),

10 The University of Texas MD Anderson Cancer Center, The University of Texas MD Anderson

11 Cancer Center

$12{ }^{4}$ Surgical Oncology, The University of Texas MD Anderson Cancer Center

$13{ }^{5}$ Division of Gastrointestinal Oncology, H. Lee Moffitt Cancer Center

$14{ }^{6}$ Department of Translational Molecular Pathology, The University of Texas MD Anderson

15 Cancer Center

16 Corresponding Author: Haoqiang Ying, MD Anderson Cancer Center, 1515 Holcombe Blvd,

17 Houston, TX 77030, Phone: 713-563-3367; E-mail: hying@mdanderson.org; and Wantong

18 Yao, MD Anderson Cancer Center, 1515 Holcombe Blvd, Houston, TX 77030. Phone: 713-

19 563-4411; E-mail: wyao22@mdanderson.org

20 Conflict of interest: The authors declare no potential conflicts of interest. 


\section{Abstract}

22 Pancreatic ductal adenocarcinoma (PDAC) is almost universally lethal. A critical unmet need exists to explore essential susceptibilities in PDAC and identify druggable targets for tumor maintenance. This is especially challenging in the context of PDAC, in which activating mutations of KRAS oncogene (KRAS*) dominate the genetic landscape. By using an inducible Kras $^{G 12 D}$-driven $p 53$ deficient PDAC mouse model (iKras model), we demonstrate that RAFMEK-MAPK signaling is the major effector for oncogenic Kras-mediated tumor maintenance. However, MEK inhibition has minimal therapeutic effect as single agent for PDAC both in vitro and in vivo. Although MEK inhibition partially downregulates the transcription of glycolysis genes, it surprisingly fails to suppress the glycolysis flux in PDAC cell, which is a major metabolism effector of oncogenic KRAS. Accordingly, In vivo genetic screen identified multiple glycolysis genes as potential targets that may sensitize tumor cells to MAPK inhibition.

33 Furthermore, inhibition of glucose metabolism with low dose 2-deoxyglucose (2DG) in combination with MEK inhibitor dramatically induces apoptosis in $\mathrm{Kras}^{\mathrm{G} 12 \mathrm{D}}$-driven PDAC cell in vitro, inhibits xenograft tumor growth and prolongs the overall survival of genetically engineered mouse PDAC model. Molecular and metabolism analyses indicate that co-targeting glycolysis and MAPK signaling results in apoptosis via induction of lethal ER stress. Together, our work suggests that combinatory inhibition of glycolysis and MAPK pathway may serve as an alternative approach to target KRAS-driven PDAC. 


\section{Introduction}

Pancreatic ductal adenocarcinoma (PDAC) is a highly aggressive malignancy with an overall 5-year survival of $<10 \%$ and projected to be the second leading cause of cancer-related death by 2030 in the United States $(1,2)$. PDAC is almost universally driven by mutationally activated KRAS, which represents the earliest and the most frequent (>90\%) genetic alteration. However, no effective inhibitors have been developed for mutant KRAS due to the lack of suitable pockets except for small molecule inhibitors targeting $\mathrm{KRAS}^{\mathrm{G} 12 \mathrm{C}}$, which is present in just $1.5 \%$ human PDAC (3-5). To date, no targeted therapy has shown any major effect in PDAC patients yet.

KRAS mutation activates several downstream signaling pathways, including, but not limited to, the RAF/MEK/MAPK, phosphatidylinositol 3-kinase (PI3K)/AKT, and RalGDS pathways (6). Consequently, multiple cellular processes are activated such as proliferation, survival and KRAS-dependent metabolism pathways $(7,8)$. However, not all of the pathways are activated by oncogenic KRAS simultaneously in any given tumor types. It is likely that a subset of KRAS surrogates play dominant roles during tumor maintenance. While several studies have established the roles of KRAS surrogates during PDAC initiation and development $(5,9,10)$, the requirement of these pathways for tumor maintenance has not been thoroughly investigated. Recently, study using genetically engineered mouse (GEM) models indicates that $\mathrm{C}-\mathrm{RAF}$ is required for the maintenance of Kras-driven lung adenocarcinomas (11). 

reprogramming in advanced tumors (14). Interestingly, recent studies have demonstrated the metabolism (17) with MAPK inhibition in PDAC treatment, underscoring the therapeutic potential of co-targeting KRAS signaling and metabolism programs. signaling, but not PI3K or RalGDS pathways, for KRAS-dependent PDAC maintenance. However, MAPK inhibition alone fails to suppress the glycolysis flux induced by oncogenic KRAS. Accordingly, genetic or pharmacological inhibition of glycolysis synergizes with MAPK inhibition to suppress PDAC growth both in vivo and in vitro. Moreover, molecular studies revealed that the synergistic effect is largely due to the induction of lethal ER stress and apoptosis. Our study identified the co-inhibition of MAPK signaling and glycolysis flux as an alternative approach to target KRAS-driven PDAC.

\section{Material and methods}

\section{$80 \quad$ Animals}

81 All animal manipulations were approved by the Animal Care and Use Committees at The

82 University of Texas, MD Anderson Cancer Center under protocol number 00001549. No 
83 patient samples were directly used in this study. TetO_Lox-Stop-Lox-Kras ${ }^{\text {G12D }}$

84 (tetO_LKras ${ }^{G 12 D}$ ), ROSA26-LSL-rtTA-IRES-GFP (ROSA_rtTA), p48-Cre and Trp53 ${ }^{L}$ strains

85 were described previously (18). Mice were fed with doxy water (Dox $2 \mathrm{~g} / \mathrm{L}$ in sucrose 20g/L)

86 starting at the 3-week-old to induce PDAC development. For drug treatment, 10-week-old mice

87 were treated by oral gavage delivery of Trametinib $(1 \mathrm{mg} / \mathrm{kg} / \mathrm{day})$, by intraperitoneal injection

88 of $2 \mathrm{DG}(1000 \mathrm{mg} / \mathrm{kg} / \mathrm{d})$ or both drugs together.

\section{Cell culture and reagents}

90 IMR90 and human PDAC cell lines HPAC, 8988T, PaTu8902, Miapaca2, DanG, S2013, and

91 PANC1 were purchased from American Type Culture Collection (ATCC). PDX148 was established from PDX tumors (19). IMR90 was grown in Eagle's Minimum Essential Medium with 10\% FBS. All human PDAC cell lines and PDX148 cells were cultured in RPMI1640 supplemented with 10\% FBS. Mouse PDAC cultures derived from the iKras/p53 and LSL-

95 Kras/p53 models were cultured in RPMI1640 supplemented with 10\% Tetracycline Negative

96 FBS (Gemini Bio Products). Trametinib, SCH772984, BKM120, GDC-0623, gemcitabine, and 97 paclitaxel were purchased from Selleckchem. 4-Phenylbutyric Acid (4-PBA) and 2DG were 98 obtained from Sigma Aldrich.

\section{Plasmids and reagents}

100 The lentiviral shRNA clones targeting mouse aldolase A and nontargeting shRNA control were

101 obtained from Sigma Aldrich in the pLKO vector. The clone IDs and sequences for shRNA are

102 listed in the Supplementary Table S2. All ORFs were cloned into pHAGE-IRES-GFP using $103 \mathrm{pENTR}^{\mathrm{TM}} / \mathrm{D}-\mathrm{TOPO}^{\mathrm{TM}}$ Cloning Kit (Thermofisher Scientific). The virus package plasmids 
104 psPAX2 (Addgene plasmid 12260) and pMD2.G (Addgene plasmid 12259) were purchased

105 from Addgene.

106 Glucose consumption and lactate production by YSI

107 Cells were seeded in 12-well plate at $\sim 30 \%$ confluence (blank wells without cells were used

108 for a baseline reading of Glucose/lactate). Forty-eight hours later, the culture medium was

109 collected from each well and spinned at $1000 \mathrm{~g}$ for $5 \mathrm{~min}$ at $4{ }^{\circ} \mathrm{C}$. $250 \mu \mathrm{l}$ supernatant was

110 transferred into 96-well plate and read on YSI (Agilent). The number of cells in the 12-well

111 plate was counted for normalization.

112 Analysis of oxygen consumption rate (OCR) and glycolytic rate (ECAR) by Seahorse XF

\section{Analyzers}

114 The determination of OCR or ECAR values was performed on XF96 analyzers. The iKras cells

115 were seeded at a density of $1 \times 10^{4}$ each well in Seahorse XF96 Cell Culture Microplate. The

116 cells were washed twice with PBS on the second day and treated as Dox ON, Dox OFF or with

117 Dox ON/TRA. After 24 h treatment, the cells were washed and cultured in Agilent Seahorse

118 XF Base Medium, and the OCR or ECAR values were detected with Seahorse XF Cell Mito

119 Stress Test following the manufacturer's instruction. After seahorse analysis, the cells in 96-

120 well plate were fixed with $4 \%$ paraformaldehyde for $15 \mathrm{~min}$, stained with DAPI and counted

121 on Operetta High-Content Imaging System. The OCR and ECAR were normalized to the cell

122 number in each well.

\section{Quantitative RT-PCR}


124 Total RNA was extracted by the Qiagen RNeasy kit following the manufacturer's instruction.

125 cDNA was generated by using the SuperScript IV First-Strand Synthesis System (Invitrogen).

126 The qRT-PCR was performed with Fast SYBR Green Master Mix (Applied Biosystems) in 96-

127 well format in StepOnePlus (Applied Biosystems). Relative expression of genes was calculated

128 by $2^{-\Delta \Delta \mathrm{CT}}$ method and normalized to beta-actin expression. All the used primers are listed in

129 Supplementary Table S3.

130 In vivo shRNA screen

131 The construction of customized shRNA library and method for shRNA screen in vivo were

132 previously described (20,21). Briefly, targeting sequences of shRNA were designed using a

133 proprietary algorithm (Cellecta) and 10 shRNA targeting each gene were included in the library.

134 The polled shRNA was cloned into the pRSI16 lentiviral vector by using chip-based

135 oligonucleotide synthesis. The oligonucleotide corresponding to each shRNA was synthesized

136 with a unique molecular barcode (18 nucleotides) for measuring representation by next-

137 generation sequencing. To perform the shRNA screen, the lentivirus package was prepared in

138 293FT cells using the second-generation packaging plasmids psPAX2 and pMD2.G. The

139 lentivirus was concentrated using ultracentrifuge at 23,000 rpm for $3 \mathrm{~h}$ and the transducing unit

140 was determined. The lentivirus infection was performed at $\sim 0.3$ transducing unit/cell with 10

$141 \mu \mathrm{g} / \mathrm{ml}$ polybrene. After puromycin selection $(2 \mu \mathrm{g} / \mathrm{ml})$ for $48 \mathrm{~h}$, cells were trypsinized, pooled

142 together and $10^{6}$ cells were washed with PBS and stored in $-80^{\circ} \mathrm{C}$ as reference. The remaining

143 cells were mixed with matrigel (1:1) and orthotopically injected at $10^{6}$ cells/mouse pancreas. 
144 The screen was conducted in triplicates and an in vivo coverage of 1000 cells/barcode was

145 guaranteed. The tumors were collected 10 days post injection and stored at $-80^{\circ} \mathrm{C}$.

\section{Extraction of tumor DNA and NGS library preparation}

147 The frozen tumors were minced to small pieces and suspended in buffer P1 (QIAGEN, $1 \mathrm{~mL}$

148 Buffer/100 mg tumor) supplemented with $100 \mu \mathrm{g} / \mathrm{mL}$ RNase A (Promega). The dissociation of

149 the tumor performed in disposable gentleMACS M tubes (Miltenyi Biotech) with the

150 gentleMACS dissociator (Miltenyi Biotec). The cell pellet was suspended in buffer P1/RNAse

151 A and lysed by adding 1/20 volume of $10 \%$ SDS (Promega). After incubating for $20 \mathrm{~min}$ at

152 room temperature, the lysates were passed 10-15 times through a 22-gauge syringe needle to

153 shear the genomic DNA. Then the genomic DNA were extracted using the Phenol-Chloroform

154 solution. The DNA pellet was finally dissolved over-night in UltraPure distilled water

155 (Invitrogen) and DNA concentration was assessed by NanoDrop 2000 (Thermo Scientific). To

156 prepare the NGS libraries, the barcodes were amplified from the equal amount of genomic

157 DNA by 2 rounds of nested PCR. The primers are list in Supplementary Table S4. The required

158 adapters for NGS were introduced in the second PCR reactions. Amplified PCR products were

159 purified using QIAquick gel extraction kit (Qiagen) and quantified using High Sensitivity DNA

160 Assay (Agilent Technologies) for the Agilent 2100 Bioanalyzer.

161 Screen data analysis

162 The shRNA screen data was analyzed as described previously $(20,21)$. Illumina base calls were

163 processed using CASAVA (v.1.8.2), and resulting reads were processed using our in-house 
164 pipeline. Following filtration and library-size normalization, reads counts in Vehicle or TRA

165 samples were compared to the reference and a Log2 fold change was calculated.

166 TEM

167 Cells were cultured in 12-well plate and washed with PBS twice before fixed with a solution

168 containing 3\% glutaraldehyde and 2\% paraformaldehyde in $0.1 \mathrm{M}$ cacodylate buffer (pH 7.3).

169 Samples were then washed in $0.1 \mathrm{M}$ sodium cacodylate buffer, treated with $0.1 \%$ Millipore-

170 filtered cacodylate buffered tannic acid, post-fixed with $1 \%$ buffered osmium and stained with

$1710.1 \%$ Millipore-filtered uranyl acetate. The samples were dehydrated in increasing

172 concentrations of ethanol and then infiltrated and embedded in LX-112 medium. The samples

173 were then polymerized in a $60^{\circ} \mathrm{C}$ oven for three days. Ultrathin sections were cut using a Leica

174 Ultracut microtome (Leica, Deerfield, IL) and then stained with uranyl acetate and lead citrate

175 in a Leica EM Stainer. The stained samples were examined in a JEM 1010 transmission

176 electron microscope (JEOL USA, Inc., Peabody, MA) using an accelerating voltage of $80 \mathrm{kV}$.

177 Digital images were obtained using an AMT imaging system (Advanced Microscopy

178 Techniques Corp., Danvers, MA).

179 Cell Viability Assay

180 Cells were plated in equal number in 24 well plates and treated with 2DG, Trametinib or

181 combination. After 4 days' treatment, cells were rinsed twice with PBS to eliminate the floating

182 cells and stained by Crystal Violet Staining Solution (0.25\% Crystal violet in $20 \%$ methanol)

183 for $20 \mathrm{~min}$. The staining solution was removed and cells were washed with water. Stained cells

184 were dried at room temperature and scanned. To quantify the relative cell numbers, cells were 
destained with $10 \%$ Acetic acid and absorbance was measured at $595 \mathrm{~nm}$ at appropriate dilutions. The Bliss Score for the combination was calculated by online tools

187 (https://synergyfinder.fimm.fi/).

\section{Annexin V-PE and 7-AAD apoptosis assay} antibody and $5 \mu \mathrm{l}$ 7-AAD were added. After staining at room temperature for $15 \mathrm{~min}$, the samples were analyzed on Gallios Flow Cytometer (Beckman Counter). The FACS data were analyzed with FlowJo.

\section{Xenograft}

199 For orthotopic xenografts, $5 \times 10^{5}$ cells suspended in $10 \mu 150 \%$ Corning Matrigel Matrix

200 (Corning)/Opti-MEM media were injected into the pancreas of NCr nude mice.

201 For Sub-Q xenografts, $1 \times 10^{6}$ cells suspended in $100 \mu 1$ Opti-MEM media were injected

202 subcutaneously into the lower flank of NCr nude mice. Animals were fed with doxy water and

203 treated with Trametinib $(1 \mathrm{mg} / \mathrm{kg} / \mathrm{d}), 2 \mathrm{DG}(1000 \mathrm{mg} / \mathrm{kg} / \mathrm{d})$ or combination. Tumor volumes and

204 body weight were measured every three days starting from Day 4 postinjection and calculated

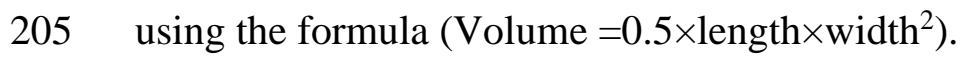




\section{RNA sequencing}

207 For the tumor samples, the mice were randomized into three groups (Kras-ON/Vehicle, Kras-

208 OFF/Vehicle, Kras ON/TRA) on the $7^{\text {th }}$ day after orthotopic injection. After 1 or 3 days of

209 treatment, the tumors were collected for RNA extraction. For the cell samples, $5 \times 10^{5} \mathrm{iKras}$ cell

210 were seeded in the $10 \mathrm{~cm}$ dish and started the treatment with vehicle, $1 \mathrm{mM} 2 \mathrm{DG}, 25 \mathrm{nM}$ TRA,

211 or combination for $24 \mathrm{~h}$. The RNA samples were collected in the TRIzol $^{\mathrm{TM}}$ Reagent

$212\left(1 \mathrm{ml} / 10 \mathrm{~cm}^{2}\right)$.

213 Total RNA was extracted using the Qiagen RNeasy kit following the manufacturer's instruction.

214 The RNA samples with a RIN score >8 were used in further analysis. RNA library preparation,

215 sequencing, raw data processing, and quality control were performed by Advanced Technology

216 Genomics Core at MD Anderson Cancer Center. Reads were mapped using Tophat and FPKM

217 values were generated with Cufflinks. The software package LIMMA (Linear Models for

218 Microarray Data) was applied to detect significantly differentially expressed genes using

219 Benjamini-Hochberg adjusted p-values.

220 Immunohistochemistry and Western Blot Analysis

221 Tissues were fixed in $10 \%$ formalin overnight and embedded in paraffin.

222 Immunohistochemical analysis was performed as described (22). Antibodies used for

223 immunohistochemistry and WB were listed in Supplementary Table S5 and Table S6.

\section{Statistical analysis}

225 To assess variance differences across various test groups, the data were analyzed using multiple

226 t-tests in GraphPad Prism. Other comparisons were performed by using the unpaired 2-tailed 
t-test. For all figures with error bars, data are presented as mean \pm SD unless otherwise stated.

228 Tumor volume and tumor-free survival results were analyzed using GraphPad Prism. The level of significance was set at for $\mathrm{p}$-value $<0.01(* *)$ or $\mathrm{p}$-value $<0.05(*)$ in all figures.

\section{Results}

\section{Active MAPK pathway is essential for PDAC maintenance}

Our previous study has established the essential role of oncogenic KRAS for PDAC

maintenance (14). To dissect the respective contributions of KRAS downstream pathways in

241 dissect the respective contributions of Kras downstream pathways for tumor maintenance (Fig.

$243 \mathrm{~S} 1 \mathrm{~A})$, functions as potent as the endogenous $\mathrm{Kras}^{G 12 D}$, to maintain xenograft tumor growth

244 following doxycycline withdrawal (Fig. 1B). In contrast, Kras ${ }^{\mathrm{G} 12 \mathrm{~V} / \mathrm{Y} 40 \mathrm{C}}$ (activates PI3K) or

$245 \mathrm{Kras}^{\mathrm{G} 12 \mathrm{~V} / \mathrm{E} 37 \mathrm{G}}$ (activates RalGDS) (Fig. S1A) was less efficient to maintain the tumor growth

246 upon extinction of the endogenous $\mathrm{Kras}^{G 12 D}$. In consistent with the in vivo observation, the

247 induction of sphere formation by $\mathrm{Kras}^{\mathrm{G} 12 \mathrm{~V} / \mathrm{T} 35 \mathrm{~S}}$ in the absence of endogenous Kras ${ }^{\mathrm{G} 12 \mathrm{D}}$ is 
248 comparable with $\mathrm{Kras}^{\mathrm{G} 12 \mathrm{~V}}$. However, the number of spheres was reduced by about $90 \%$ if

$249 \mathrm{Kras}^{\mathrm{G} 12 \mathrm{~V} / \mathrm{Y} 40 \mathrm{C}}$ or Kras ${ }^{\mathrm{G} 12 \mathrm{~V} / \mathrm{E} 37 \mathrm{G}}$ was expressed in iKras cells in the absence of doxycycline (Fig.

250 1C-D). Therefore, our data implicates the dominant role of RAF/MAPK signaling for KRAS-

251 driven PDAC maintenance.

252 To further dissect the KRAS effector pathways in advanced tumors, we conducted gain of

253 function experiments using several constitutively activated mutants for the key effectors in

254 MAPK, PI3K or RalGDS pathway (Fig. S1B-C). Consistent with our findings with the KRAS

255 effector mutants, our data indicated that the constitutively activated $\mathrm{C}-\mathrm{Raf}^{\mathrm{W} 22}, \mathrm{BRAF}^{\mathrm{V} 600 \mathrm{E}}$, and

$256 \operatorname{MEK}^{\mathrm{DD}}$ (25) were as efficient as mutant Kras to sustain tumor sphere formation in vitro and

257 maintain tumor growth in vivo in the absence of doxycycline (Fig. 1E-G). In contrast,

258 constitutive active PI3K $\mathrm{K}^{\mathrm{H} 1047 \mathrm{R}}$ was less competent to support tumor sphere growth and induced

259 much delayed tumor formation compared to the constitutive active mutants of the RAF/MAPK

260 pathway components (Fig. 1F-G and S1D). Additionally, in the absence of doxycycline, iKras

261 cells expressing Myr-AKT or constitutive active RalGDS (RalA ${ }^{\mathrm{Q} 75 \mathrm{~L}}$ or $\mathrm{RalB}^{\mathrm{Q} 72 \mathrm{~L}}$ ) failed to

262 exhibit tumorigenic activity in vitro or in vivo (Fig. 1F-G and S1D). Together, our data

263 indicates that RAF-MAPK signaling is the major pathway for KRAS-mediated PDAC

264 maintenance.

265 Inhibition of MAPK pathway fails to recapitulate glycolysis inhibition upon

266 KRAS inactivation.

267 Despite the essential role of MAPK pathway in PDAC maintenance, blocking MAPK

268 signaling with MEK inhibitors has been shown to exert marginal impact on tumor growth or 
overall survival in both preclinical models and PDAC patients (12,13,26-28). Although the feedback activation of multiple RTKs and their downstream PI3K pathway has been shown to mediate the resistance to MPAK inhibition in KRAS-driven PDAC (12,13), co-targeting in preclinical model (12). In an effort to further explore potential MAPK co-inhibition targets, we leveraged the unique ability of the iKras model to genetically extinct oncogenic Kras in advanced tumors and conducted global transcriptomic analysis to identify KRAS downstream analysis was performed in orthotopic xenograft tumors established with primary tumor lines from the iKras/p53 model to compare the transcriptomic profiles following KRAS extinction or MEK inhibitor (MEKi) treatment for 1 or 3 days (Fig. 2A-B).

The principal component analysis (PCA) revealed high concordance among the three biological replicates within each treatment group. Notably, a progressing shift was observed in

Dox OFF 1-day, and 3-day tumors compared to ON Dox tumors, indicating the time-dependent transcriptomic change following KRAS extinction. However, such gradual expression changes were not observed in Trametinib-treated tumors (Fig. 2C), prompting the hypothesis that the pathways downstream of oncogenic KRAS that are not affected by treatment with MEKi could serve as coextinction targets that may cooperate with MEKi to recapitulate the impact of KRAS extinction. To this end, we conducted Ingenuity Pathway Analysis (IPA) on the differentially expressed genes in the Dox OFF group or the Trametinib treated group while compared with the Dox ON group. Half of the top-ten differentially expressed pathways preferentially 
enriched in OFF Dox tumors are associated with chromosol replication or cell cycle control

302 iKras/p53 model. Upon KRAS inactivation, glucose consumption or lactate production in the

303 medium was dramatically downregulated, which is further supported by the decrease of

304 extracellular acidification rate (ECAR) as measured with Seahorse. Surprisingly, no significant

305 decrease in glucose consumption or lactate production was detected when the cells were treated

306 with Trametinib for 2 days (Fig. 2F-G). Moreover, Seahorse analysis showed that Trametinib

307 treatment lead to a mild induction of ECAR compared with the iKras/p53 tumor cells grown in

308 the presence of doxycycline (Fig. 2H and Fig. S2C-D). Accordingly, MEK inhibition also

309 failed to suppress the glucose uptake and lactate production in human PDAC cell lines (Fig.

310 S2E-F). BKM120, a selective PI3K inhibitor, was able to reverse the AKT activation induced 
311 by Trametinib treatment and impaired the glucose consumption and lactate production (Fig. 2I

312 and S2G), indicating that the sustaining of glycolysis activity upon MEK inhibition is PI3K-

313 AKT-dependent in iKras cell. Together, our transcriptomic analysis and biochemical analysis

314 indicate that MEK inhibition alone is not sufficient to suppress the KRAS-mediated glycolysis

315 flux in PDAC cells, likely due to the feedback activation of PI3K signaling.

316 Pooled shRNA library screening indicates glycolysis inhibition sensitizes iKras

317 cells to MEK inhibition

318 To identify the metabolism genes that may sensitize Kras-driven PDAC cell to MEK

319 inhibition upon depletion, we performed a pool-based in vivo loss-of-function screen in the

320 orthotopic xenograft model as previously described (21). A customized bar-coded shRNA

321 library comprising 3,400 shRNAs targeting 340 metabolism genes, including those KRAS-

322 dependent metabolism genes (14) was packaged into lentivirus and infected iKras/p53 mouse

323 PDAC cells. Orthotopic xenograft tumors were established in the nude mice and were treated

324 with vehicle or Trametinib for 10 days before collection for next-generation sequencing. The

$325 \log$ fold change $(\log F C)$ of bar-coded shRNA in the control or Trametinib-treated xenograft

326 was calculated by comparison with reference group which was composed of library infected

327 cells collected before orthotopic injection (Fig. 3A). In both control and Trametinib-treated

328 xenograft, the positive control shRNA targeting PMSA1 and RPL30 are most depleted

329 compared with the reference which indicates a high reliability of our screen system. Using

$330 \log \mathrm{FC}=-1$ as a cut-off value, we obtained 36 candidate genes that were selectively depleted in

331 MEKi-treated xenograft tumors compared to the control untreated ones (Supplementary Table 
332 S1). The depleted genes were most enriched in the glycolysis pathway (Fig. 3B), including

333 Aldoa, Gapdhs, Hk2, Eno1 and PfkP (Fig. 3C-D).

334 To validate the hits, we knocked down Aldoa in the iKras/p53 PDAC cells with shRNA

335 (Fig. 3E). While knockdown of Aldoa itself has a limited effect on the cell growth, Aldoa-

336 depleted cells are more sensitive to MEK inhibition compared with control cells, approximating

337 the inhibitory effect on cell growth following KRAS inactivation (Fig. 3F-G). Together, our

338 data suggests that glycolysis inhibition may sensitize the KRAS-driven PDAC cells to MEK

339 inhibition.

340 Glycolysis inhibition with 2DG synergizes with MEK inhibition to induce PDAC

341 cell apoptosis

342 To test if pharmacological inhibition of glycolysis will synergize with MEK inhibition in

343 KRAS-driven PDAC cells, we combined a well-known glycolysis inhibitor 2-deoxy- glucose

344 (2DG) with Trametinib to treat iKras/p53 PDAC cells. While either 2DG or Trametinib

345 treatment alone failed to significantly suppress cell growth, the combination dramatically

346 decreased the proliferation of mouse PDAC cells from the iKras/p53 and LSL-Kras/p53 models,

347 as well as human PDAC cell lines, including HPAC and Patu8902 cells (Fig. 4A-B).

348 Interestingly, no synergistic effect was observed in normal lung fibroblast IMR90 (Fig. 4A-B),

349 implicating a therapeutic window for such combination therapy. To evaluate the synergy over

350 a broad range of 2DG and Trametinib concentration, we computed the Bliss independence

351 score for Trametinib and 2DG combination in PDAC cell lines. The strong synergistic effect

352 was indicated by a high positive score (=29.014) in the iKras/p53 PDAC cells but not in IMR90 
cell (Bliss score=0.57) (Fig. 4C-D). The synergy between 2DG and Trametinib was also

\section{DG and MEK inhibition synergistically induces apoptosis through ER stress}

To gain molecular insight into the mechanisms underlying the synergy between 2DG and $\mathrm{p}<0.01$, a total of 850 up-regulated and 310 down-regulated genes were identified from three treatment groups compared to vehicle control group (Fig. S4A and S4B). IPA analysis of the 

treatment group, including 526 up- and 210 down-regulated genes. The top two enriched pathways are autophagosome maturation and unfold protein response (UPR), which are not among the top enriched pathways in the single treatment groups, implicating the relevance to the apoptosis induced by the combination therapy (Fig. 5C). treated iKras/p53 tumor cells (Fig. 5D), a morphology change indicating the induction of UPR.

The activation of UPR was further supported by the drastic upregulation of ER stress markers, including ATF4, ATF6 and BIP, following 2DG/Trametinib treatment compared with vehicle control of single treatment groups (Fig. 5E-F). Moreover, CHOP, a well-known apoptosis activator downstream of UPR, along with cleaved caspase 3, was also specifically upregulated by the combination treatment in iKras/p53 tumor cells (Fig. 5E-F), indicating 2DG and MEK inhibition results in lethal ER Stress in PDAC cells. Interestingly, no induction of ER stress markers or cleaved caspase 3 was observed in 2DG/Trametinib-treated IMR90 cells (Fig. S4C). To further validate if unfold protein response is responsible for the induction of apoptosis by

389 the combined 2DG and Trametinib treatment, cells were treated with the chemical chaperon

390 PBA to decrease the UPR. As expected, PBA inhibited the induction of multiple ER-stress 391 markers, such as BIP, ATF4, and phosphor-EIF2 $\alpha$. Importantly, PBA treatment dramatically 392 decreased the expression of CHOP, prevented caspase-3 cleavage and suppressed apoptosis 393 induced by 2DG and Trametinib treatment (Fig. 5G-H), indicating the cell death induced by 394 the combination treatment is mediated by hyper-activation of UPR. 


\section{Trametinib in combination with 2DG exhibits antitumor activity in vivo}

Next, we sought to evaluate the therapeutic potential of the combination in vivo. Briefly,

397 the mouse iKras/p53 PDAC cells or PDX-derived human PDAC cells were injected

398 subcutaneously into the immune-deficient mice. Tumor-bearing mice were treated with 2DG

$399(1000 \mathrm{mg} / \mathrm{kg} / \mathrm{d})$, Trametinib $(1 \mathrm{mg} / \mathrm{kg} / \mathrm{d})$ or combination. Compared with single treatment group,

400 combination group exhibited significant decrease in tumor size for both human and mouse

401 PDAC (Fig. 6A-B). Moreover, we also evaluated the effect of the combination in the GEMM

402 (iKras/p53 $\left.3^{\mathrm{L} /+}\right)$. After 7 weeks' induction of $\mathrm{KRAS}^{\mathrm{G} 12 \mathrm{D}}$ expression with doxycycline, a time

403 point previously showed to induce invasive carcinoma (14), the mice were randomized into

404 four groups, including Vehicle control, Trametinib, 2DG, and Combo groups. While single

405 treatment failed to elicit anti-tumor effect, combination treatment significantly prolonged

406 overall survival (Fig. 6C). Immunohistochemistry revealed that the percentage of ki67 positive

407 cells was significantly decreased in the combo-treated tumors compared to the single treatment

408 groups, indicating inhibition of tumor cell proliferation (Fig. 6D and 6F). More importantly,

409 BIP expression and percentage of cleaved Caspase-3 positive cells were significantly

410 upregulated in the tumors treated with 2D/Trametinib combination (Fig. 6D and 6E),

411 supporting the induction of UPR-related apoptosis.

\section{Discussion}

As the major surrogates of KRAS signaling, the roles of MAPK and PI3K pathway in

414 KRAS-driven tumors have been extensively studied. Mutations of BRAF, a dominant mediator

415 for oncogenic KRAS signaling to activate MAPK signaling (30), were found to be mutually 
416 exclusive with the KRAS mutations in PDAC (5). Pancreatic-specific expression of oncogenic

$417 \mathrm{Braf}^{V 600 E}$ is sufficient to induce intraepithelial neoplasia (PanIN) lesions and invasive PDA in

418 the autochthonous models (9). Moreover, CRAF has been shown to be essential for

419 development of KRAS-driven non-small cell lung carcinoma $(31,32)$, further supporting the

420 central role of MAPK pathway in KRAS-driven tumorigenesis. On the other hand, PI3K and

421 PDK1 have also been shown to be critical effectors downstream of oncogenic Kras in

422 mediating cell plasticity and PDAC development (10). These data indicate that both MPAK

423 and PI3K pathways are essential for KRAS-mediated tumor initiation. However, the

424 requirement of these KRAS surrogates in advanced tumors has been less clear.

425 Recent study showed that ablation of CRAF expression leads to significant tumor

426 regression in advanced tumors driven by $\mathrm{KRAS}^{\mathrm{G} 12 \mathrm{~V}} / \mathrm{Trp} 53$ mutations (11), underscoring the

427 requirement of MAPK pathway for tumor maintenance. Here our data provide additional

428 evidences that MAPK pathway is necessary and sufficient for PDAC maintenance whereas

429 PI3K activation is less competent to sustain tumor growth by itself, supporting the need to

430 target MAPK pathway in KRAS-driven tumors. However, it has been well documented that

431 targeting MAPK alone failed to elicit therapeutic benefit in KRAS-driven tumors, likely due

432 to the feedback activation of PI3K pathway (12,33). Although co-targeting MAPK and PI3K

433 was able to induce tumor regression and prolong survival in PDAC GEMM $(12,13)$, the

434 combination is too toxic to be tolerant in human patients (34). Previous studies have identified

435 reactivation of multiple RTKs as a prominent mechanism of adaptive resistance to MEK

436 inhibition in KRAS-driven tumors $(13,33,35)$. However, co-targeting multiple RTKs is 
437 difficult to achieve therapeutically, pointing to the need to identify additional strategies to 438 target PI3K or its downstream effector pathways.

439 In this study, we identified the potential role of PI3K-mediated glycolysis in the adaptive 440 resistance to MEK inhibition in KRAS-driven PDAC. Co-targeting MAPK pathway and 441 glycolysis with Trametinib and 2DG combination synergistically induces apoptosis in tumor 442 cells both in vivo and in vitro. In line with our findings, recent study in BRAF-driven melanoma 443 showed that glycolysis inhibitors were able to potentiate the effects of Braf inhibitor (36). 444 Although MAPK signaling has been shown to mediate the transcription of multiple glycolysis genes downstream of oncogenic KRAS $(14,17)$, here we showed that feedback activation of 446 PI3K pathway is sufficient to maintain glycolysis flux in KRAS-driven tumors following

447 MAPK inhibition. PI3K has been shown to be a master regulator for the transcription of glucose 448 transporters (37). PI3K can also activate glycolysis at post-translational level by controlling 449 the cytoskeleton remodeling and thus relieving the sequestration of glycolysis enzymes (38).

450 Whether such mechanisms are also involved in the feedback activation of glycolysis upon 451 MEK inhibition in KRAS-driven tumor cells remains to be elucidated.

453 PDAC cells following the inhibition of MAPK signaling. Blocking glycolysis with 2DG in 454 combination with MAPK inhibition leads to induction of apoptosis. 2DG, a derivative of 455 glucose, could be phosphorylated to 2DG-6-phosphate in cell. The accumulated 2DG-6456 phosphate inhibits hexokinase in a noncompetitive manner (39) and can lead to the inhibition 457 of multiple anabolic processes branched from glycolysis pathway. It's possible that the synergy 
between 2DG and MAPK inhibition is due the blockade of multiple glucose-dependent metabolism pathways. Among them, 2DG has been shown to impair the pentose-phosphate pathway (PPP) and dramatically decreases R5P level (40). Interestingly, recent study has shown that the activation of PPP-mediated ribose metabolism is critical for the adaptation to the inhibition of KRAS signaling in PDAC cells (17). In addition, 2DG treatment has been shown to induce ER stress, likely due to its impact on HBP or mannose metabolism $(41,42)$.

While UPR is considered as a survival mechanism to maintain protein homeostasis, excessive ER stress will result in cell death $(43,44)$. Here, we provide evidence that the combination of 2DG and MAPK inhibition dramatically amplified the ER stress in PDAC cells which results in apoptosis. This is supported by our finding that the cell death was partially rescued with chemical chaperon that mitigates the UPR. Therefore, our data indicates that the maintenance of protein homeostasis is critical for the survival of KRAS-driven PDAC cells upon the inhibition of MAPK signaling. It will be interesting to evaluate whether targeting additional regulators of protein homeostasis may also sensitize PDAC cells to MAPK inhibition. and MAPK has an alternative approach to treat KRAS-driven PDAC.

\section{Acknowledgment}

476 We thank the Institute for Applied Cancer Science for sharing reagents. We would like to thank

477 Small Animal Imaging Facility, Histopathology Core, High Resolution Electron Microscopy

478 Facility, the Flow Cytometry and Cellular Imaging Core at The University of Texas MD 
479 Anderson Cancer Center, and the Veterinary Medicine Department at MD Anderson (Cancer

480 Center Support Grant CA016672). We thank Costas A. Lyssiotis for his suggestion and

481 supports on the cell metabolism analysis. The research was supported by the National Cancer

482 Institute (NCI) grant R01CA214793 and NCI P01 grant P01CA117969 to HY, and the

483 Pancreatic Cancer Action Network-American Association for Cancer Research Pathway to

484 Leadership Award to WY. 


\section{References}

1. Rahib L, Smith BD, Aizenberg R, Rosenzweig AB, Fleshman JM, Matrisian LM. Projecting cancer incidence and deaths to 2030: the unexpected burden of thyroid, liver, and pancreas cancers in the United States. Cancer Res 2014;74:2913-21

489 2. Siegel RL, Miller KD, Jemal A. Cancer statistics, 2020. CA Cancer J Clin 2020;70:7-30

490 3. Cox AD, Fesik SW, Kimmelman AC, Luo J, Der CJ. Drugging the undruggable RAS: Mission possible? Nat Rev Drug Discov 2014;13:828-51

492 4. Canon J, Rex K, Saiki AY, Mohr C, Cooke K, Bagal D, et al. The clinical KRAS(G12C) inhibitor AMG 510 drives anti-tumour immunity. Nature 2019;575:217-23

494 5. Witkiewicz AK, McMillan EA, Balaji U, Baek G, Lin WC, Mansour J, et al. Whole-exome sequencing of pancreatic cancer defines genetic diversity and therapeutic targets. Nature communications 2015;6:6744

6. Downward J. Targeting RAS signalling pathways in cancer therapy. Nature reviews Cancer 2003;3:11-22

7. Bryant KL, Mancias JD, Kimmelman AC, Der CJ. KRAS: feeding pancreatic cancer proliferation. Trends Biochem Sci 2014;39:91-100

8. Rodriguez-Viciana P, Warne PH, Khwaja A, Marte BM, Pappin D, Das P, et al. Role of phosphoinositide 3-OH kinase in cell transformation and control of the actin cytoskeleton by Ras. Cell 1997;89:457-67

9. Collisson EA, Trejo CL, Silva JM, Gu S, Korkola JE, Heiser LM, et al. A central role for RAF-->MEK->ERK signaling in the genesis of pancreatic ductal adenocarcinoma. Cancer discovery 2012;2:685-93

10. Eser S, Reiff N, Messer M, Seidler B, Gottschalk K, Dobler M, et al. Selective requirement of PI3K/PDK1 signaling for Kras oncogene-driven pancreatic cell plasticity and cancer. Cancer Cell 2013;23:406-20

11. Sanclemente M, Francoz S, Esteban-Burgos L, Bousquet-Mur E, Djurec M, Lopez-Casas PP, et al. c-RAF Ablation Induces Regression of Advanced Kras/Trp53 Mutant Lung Adenocarcinomas by a Mechanism Independent of MAPK Signaling. Cancer Cell 2018;33:217-28 e4

12. Alagesan B, Contino G, Guimaraes AR, Corcoran RB, Deshpande V, Wojtkiewicz GR, et al. Combined MEK and PI3K inhibition in a mouse model of pancreatic cancer. Clin Cancer Res 2015;21:396-404

13. Pettazzoni P, Viale A, Shah P, Carugo A, Ying H, Wang H, et al. Genetic events that limit the efficacy of MEK and RTK inhibitor therapies in a mouse model of KRAS-driven pancreatic cancer. Cancer research 2015;75:1091-101

14. Ying H, Kimmelman AC, Lyssiotis CA, Hua S, Chu GC, Fletcher-Sananikone E, et al. Oncogenic Kras maintains pancreatic tumors through regulation of anabolic glucose metabolism. Cell 2012;149:656-70

15. Bryant KL, Stalnecker CA, Zeitouni D, Klomp JE, Peng S, Tikunov AP, et al. Combination of ERK and autophagy inhibition as a treatment approach for pancreatic cancer. Nat Med 2019;25:628-40 elicited by RAF-->MEK-->ERK inhibition suggests a treatment strategy for RAS-driven cancers. Nat Med 2019;25:620-7 
17. Santana-Codina N, Roeth AA, Zhang Y, Yang A, Mashadova O, Asara JM, et al. Oncogenic KRAS supports pancreatic cancer through regulation of nucleotide synthesis. Nat Commun 2018;9:4945

18. Chauhan SC, Ebeling MC, Maher DM, Koch MD, Watanabe A, Aburatani H, et al. MUC13 mucin augments pancreatic tumorigenesis. Molecular cancer therapeutics 2012;11:24-33

19. Auciello FR, Bulusu V, Oon C, Tait-Mulder J, Berry M, Bhattacharyya S, et al. A Stromal LysolipidAutotaxin Signaling Axis Promotes Pancreatic Tumor Progression. Cancer discovery 2019

20. Yao W, Rose JL, Wang W, Seth S, Jiang H, Taguchi A, et al. Syndecan 1 is a critical mediator of macropinocytosis in pancreatic cancer. Nature 2019;568:410-4

21. Carugo A, Genovese G, Seth S, Nezi L, Rose JL, Bossi D, et al. In Vivo Functional Platform Targeting Patient-Derived Xenografts Identifies WDR5-Myc Association as a Critical Determinant of Pancreatic Cancer. Cell Rep 2016;16:133-47

22. Aguirre AJ, Bardeesy N, Sinha M, Lopez L, Tuveson DA, Horner J, et al. Activated Kras and Ink4a/Arf deficiency cooperate to produce metastatic pancreatic ductal adenocarcinoma. Genes Dev 2003:17:3112-26

23. White MA, Nicolette C, Minden A, Polverino A, Van Aelst L, Karin M, et al. Multiple Ras functions can contribute to mammalian cell transformation. Cell 1995;80:533-41

24. Fiordalisi JJ, Holly SP, Johnson RL, 2nd, Parise LV, Cox AD. A distinct class of dominant negative Ras mutants: cytosolic GTP-bound Ras effector domain mutants that inhibit Ras signaling and transformation and enhance cell adhesion. J Biol Chem 2002;277:10813-23

25. Boehm JS, Zhao JJ, Yao J, Kim SY, Firestein R, Dunn IF, et al. Integrative genomic approaches identify IKBKE as a breast cancer oncogene. Cell 2007;129:1065-79

26. Infante JR, Somer BG, Park JO, Li CP, Scheulen ME, Kasubhai SM, et al. A randomised, doubleblind, placebo-controlled trial of trametinib, an oral MEK inhibitor, in combination with gemcitabine for patients with untreated metastatic adenocarcinoma of the pancreas. Eur J Cancer

27. Van Cutsem E, Hidalgo M, Canon JL, Macarulla T, Bazin I, Poddubskaya E, et al. Phase I/II trial of pimasertib plus gemcitabine in patients with metastatic pancreatic cancer. Int J Cancer 2018;143:2053-64

28. Kasuga A, Nakagawa K, Nagashima F, Shimizu T, Naruge D, Nishina S, et al. A phase I/Ib study of trametinib (GSK1120212) alone and in combination with gemcitabine in Japanese patients with advanced solid tumors. Invest New Drugs 2015;33:1058-67

29. Kwong LN, Costello JC, Liu H, Jiang S, Helms TL, Langsdorf AE, et al. Oncogenic NRAS signaling differentially regulates survival and proliferation in melanoma. Nat Med 2012;18:1503-10 Cseh B, Doma E, Baccarini M. "RAF" neighborhood: protein-protein interaction in the Raf/Mek/Erk pathway. FEBS letters 2014;588:2398-406

31. Karreth FA, Frese KK, DeNicola GM, Baccarini M, Tuveson DA. C-Raf is required for the initiation of lung cancer by K-Ras(G12D). Cancer Discov 2011;1:128-36

32. Blasco RB, Francoz S, Santamaria D, Canamero M, Dubus P, Charron J, et al. C-Raf, but not B-Raf, is essential for development of K-Ras oncogene-driven non-small cell lung carcinoma. Cancer Cell 2011;19:652-63

33. Manchado E, Weissmueller S, Morris JPt, Chen CC, Wullenkord R, Lujambio A, et al. A combinatorial strategy for treating KRAS-mutant lung cancer. Nature 2016;534:647-51 
34. Do K, Speranza G, Bishop R, Khin S, Rubinstein L, Kinders RJ, et al. Biomarker-driven phase 2 study of MK-2206 and selumetinib (AZD6244, ARRY-142886) in patients with colorectal cancer. Invest New Drugs 2015;33:720-8

35. Fedele C, Ran H, Diskin B, Wei W, Jen J, Geer MJ, et al. SHP2 Inhibition Prevents Adaptive Resistance to MEK Inhibitors in Multiple Cancer Models. Cancer Discov 2018;8:1237-49

36. Parmenter TJ, Kleinschmidt M, Kinross KM, Bond ST, Li J, Kaadige MR, et al. Response of BRAFmutant melanoma to BRAF inhibition is mediated by a network of transcriptional regulators of glycolysis. Cancer Discov 2014;4:423-33

37. Hoxhaj G, Manning BD. The PI3K-AKT network at the interface of oncogenic signalling and cancer metabolism. Nat Rev Cancer 2020;20:74-88

38. Hu H, Juvekar A, Lyssiotis CA, Lien EC, Albeck JG, Oh D, et al. Phosphoinositide 3-Kinase Regulates Glycolysis through Mobilization of Aldolase from the Actin Cytoskeleton. Cell 2016;164:433-46

39. Chen W, Gueron M. The inhibition of bovine heart hexokinase by 2-deoxy-D-glucose-6phosphate: characterization by 31P NMR and metabolic implications. Biochimie 1992;74:867-73

40. Ralser M, Wamelink MM, Struys EA, Joppich C, Krobitsch S, Jakobs C, et al. A catabolic block does not sufficiently explain how 2-deoxy-D-glucose inhibits cell growth. Proc Natl Acad Sci U S A 2008;105:17807-11

586 41. Xi H, Kurtoglu M, Liu H, Wangpaichitr M, You M, Liu X, et al. 2-Deoxy-D-glucose activates autophagy via endoplasmic reticulum stress rather than ATP depletion. Cancer Chemother Pharmacol 2011;67:899-910

42. Ishino K, Kudo M, Peng WX, Kure S, Kawahara K, Teduka K, et al. 2-Deoxy-d-glucose increases GFAT1 phosphorylation resulting in endoplasmic reticulum-related apoptosis via disruption of protein N-glycosylation in pancreatic cancer cells. Biochem Biophys Res Commun 2018;501:668-

593 43. Sano R, Reed JC. ER stress-induced cell death mechanisms. Biochim Biophys Acta 2013;1833:346070

595 44. Szegezdi E, Logue SE, Gorman AM, Samali A. Mediators of endoplasmic reticulum stress-induced apoptosis. EMBO Rep 2006;7:880-5 

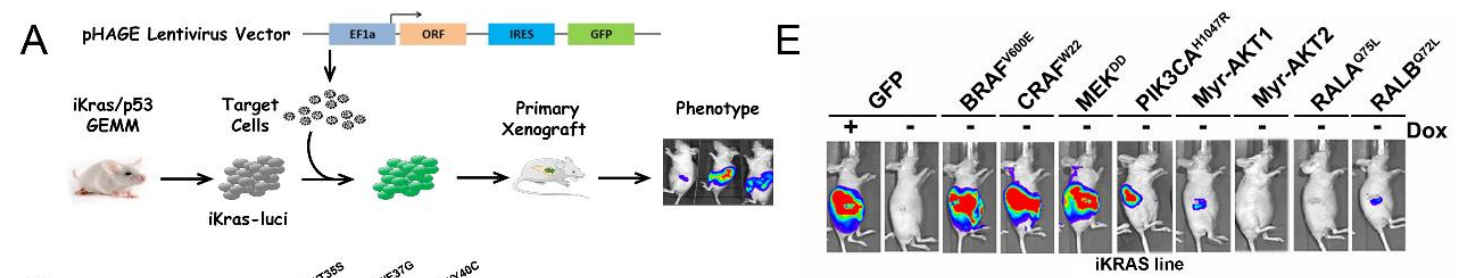

B
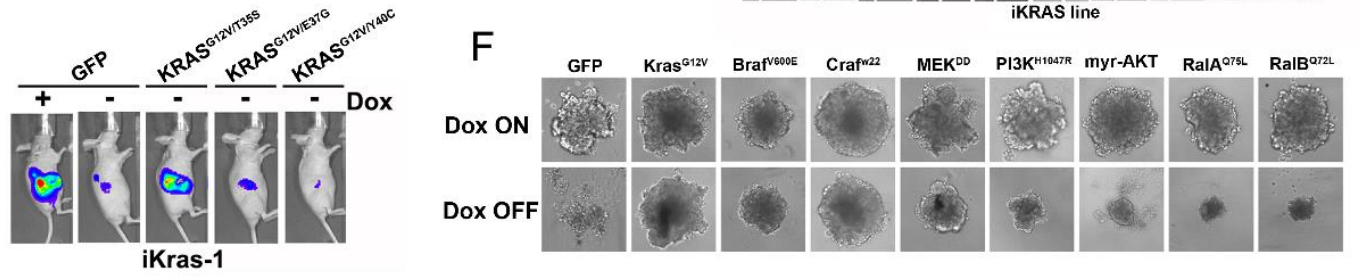

C

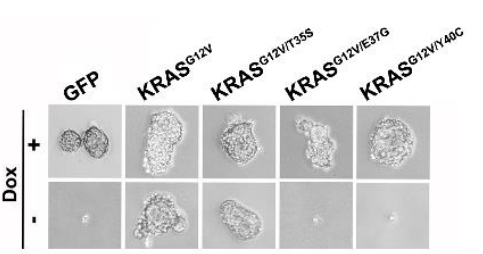

G

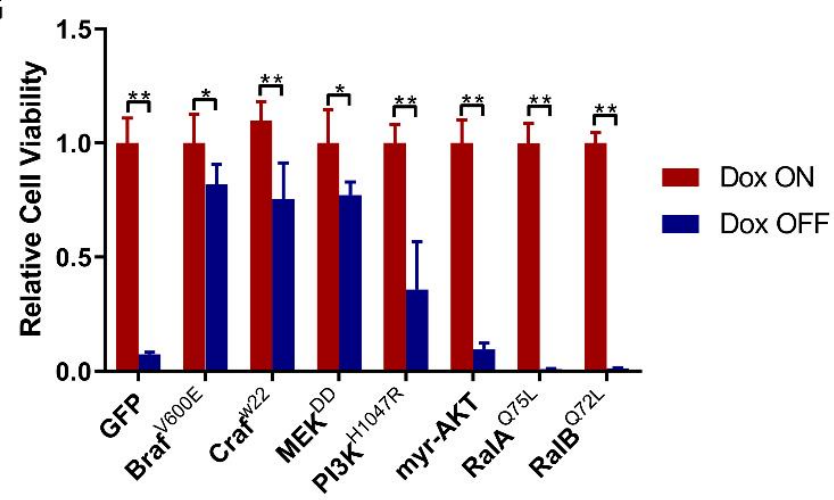

599 Figure 1. Active MAPK pathway is essential for PDAC maintenance

600 (A) Schematic diagram of investigating KRAS downstream surrogates in PDAC maintenance. The

601 iKras cell was infected with lentivirus to overexpress the KRAS surrogates, sorted and orthotopically

602 injected into nude mouse. Tumorigenesis was observed by bioluminescence imaging. (B)

603 Tumorigenesis of iKras cells with $\mathrm{Kras}^{\mathrm{G} 12 \mathrm{~V} / \mathrm{T} 35 \mathrm{~S}}$, Kras ${ }^{\mathrm{G} 12 \mathrm{~V} / \mathrm{E} 37 \mathrm{G}}$ or $\mathrm{Kras}^{\mathrm{G} 12 \mathrm{~V} / \mathrm{Y} 40 \mathrm{C}}$ overexpression by

604 bioluminescence imaging. (C) Sphere formation of iKras cell with Kras ${ }^{\mathrm{G} 12 \mathrm{~V} / \mathrm{T} 35 \mathrm{~S}}$, Kras ${ }^{\mathrm{G} 12 \mathrm{~V} / \mathrm{E} 37 \mathrm{G}}$,

$605 \mathrm{Kras}^{\mathrm{G} 12 \mathrm{~V} / \mathrm{Y} 40 \mathrm{C}}$ overexpression in the low-attached plate. (D) Quantification of sphere number in $\mathrm{C}(\mathrm{n}=3$,

606 Mean \pm SD). (E) Tumorigenesis of mouse PDAC cells with constitutively active KRAS downstream

607 surrogates by bioluminescence imaging. (F) Sphere formation of iKras PDAC cells with constitutively

608 active KRAS downstream surrogates in low attached plate. (G) Quantification of spheres in F by cell

609 viability assay $(n=3$, Mean \pm SD). 
A

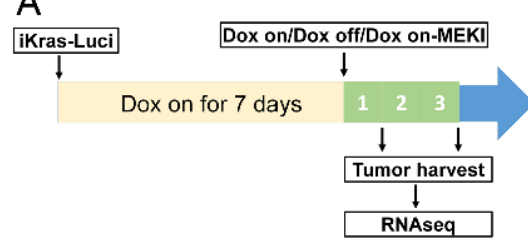

D
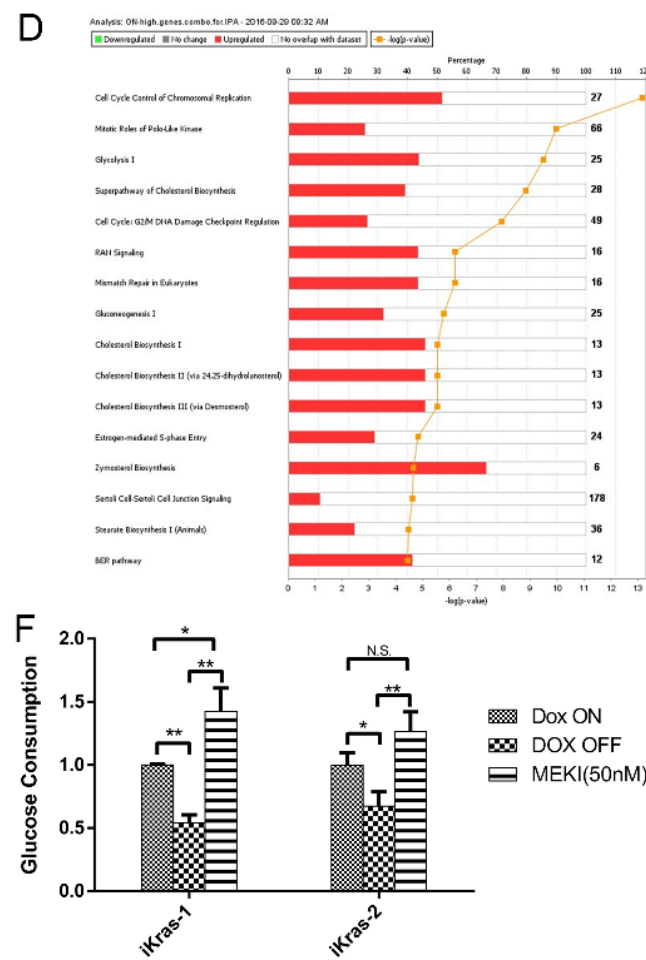

$\mathrm{H}$
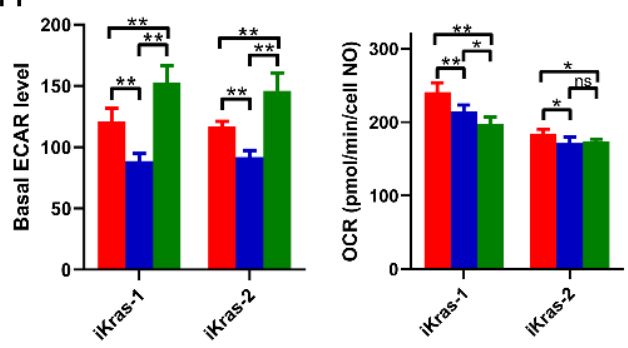

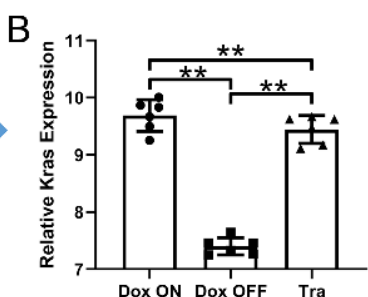

$\mathrm{E}$

Glycolysis/glucagon

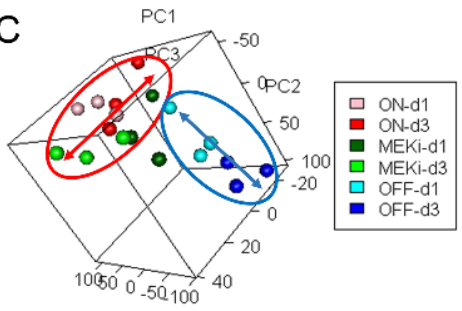

Cholesterol Biosynthesis
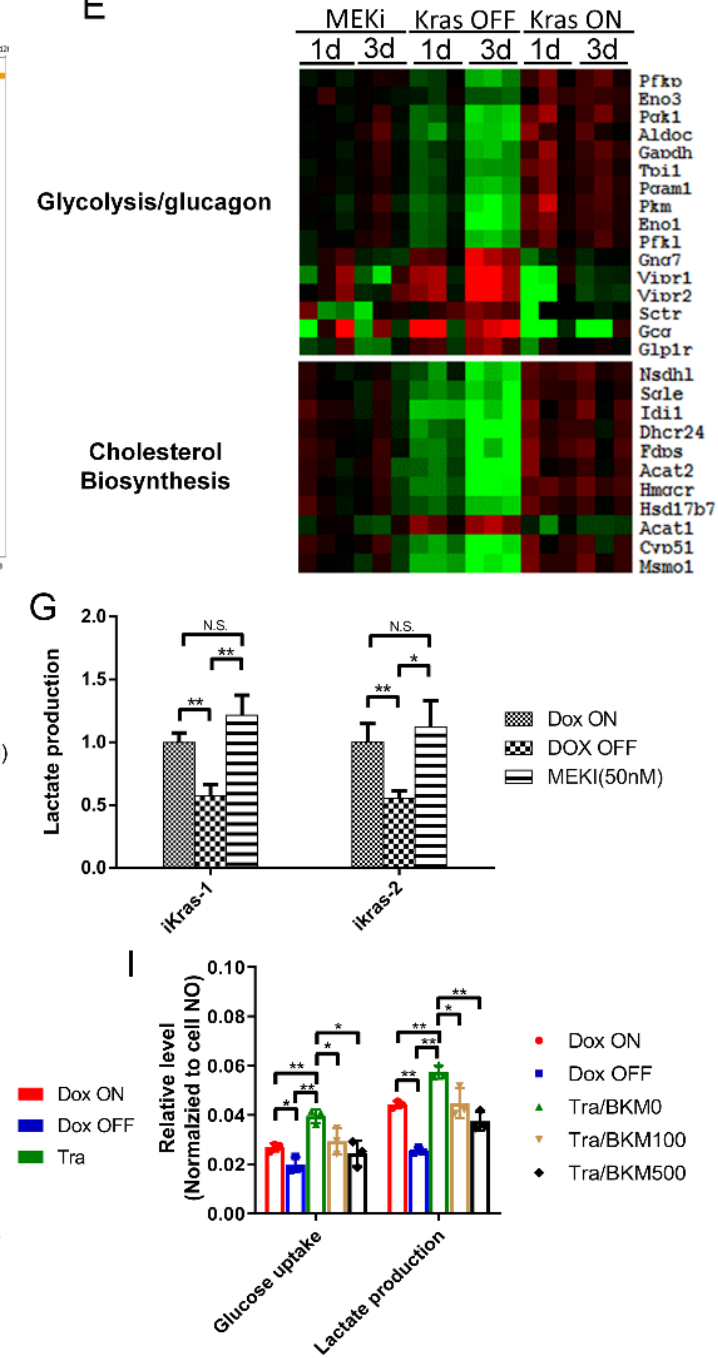

\section{Figure 2. MEK inhibition fails to recapitulate glycolysis inhibition by Kras inactivation.}

(A) Schematic illustration of transcriptomic analysis between xenograft upon Dox ON, Dox OFF or TRA treatment in vivo. Seven days post orthotopic injection of iKras cells, the mice were randomized into three group (Dox ON, Dox OFF or treated with $1 \mathrm{mg} / \mathrm{kg} /$ day TRA) and xenograft tumors were collected after 1- or 3-days treatment. (B) Kras expression in the xenograft upon Dox ON, Dox OFF or TRA treatment by RNA-seq ( $n=5$, Mean $\pm S D$ ). (C) PCA analysis of transcriptome changes between Dox ON, Dox OFF or TRA treatment. (D) IPA pathway analysis of differentially expressed genes between Dox OFF and TRA treatment. (E) Heatmap of the representative differentially expressed genes in respective pathway. (F-G) Glucose consumption and lactate production of iKras cells with Dox ON, Dox OFF or TRA treatment by YSI $(n=3$, Mean \pm SD). Expression validation of genes involved in glycolysis by qPCR $(n=3$, Mean \pm SD). (H) Basal ECAR or OCR value of iKras cells with Dox ON, 
623 Dox OFF or TRA treatment by Seahorse ( $n=4$, Mean \pm SD). (I) Glucose and lactate concentration in 624 the medium of iKras cell was measured upon treatment (TRA: 25nM; BKM120: $100 \mathrm{nM}$ or $500 \mathrm{nM}$ ) 625 for $48 \mathrm{~h}$. The glucose consumption and lactate production were normalized based on cell number $(\mathrm{n}=3$, 626 Mean \pm SD).

627 
A

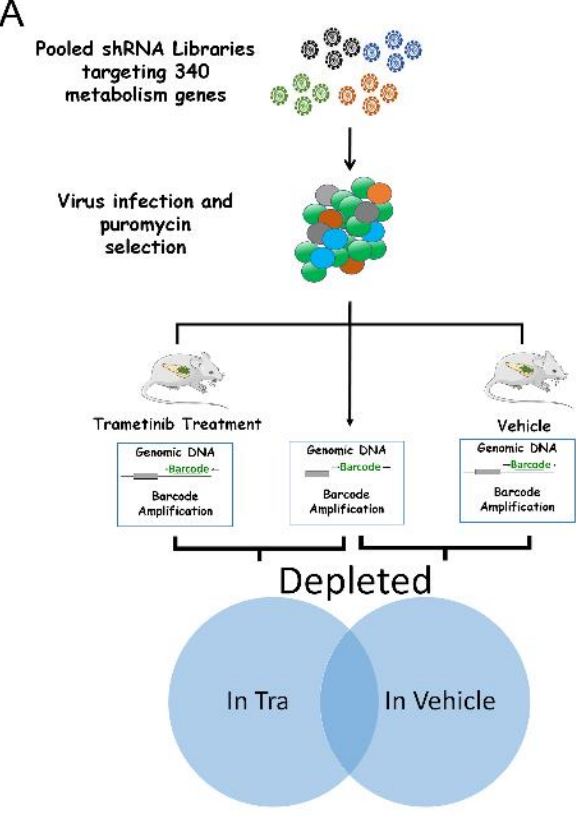

B

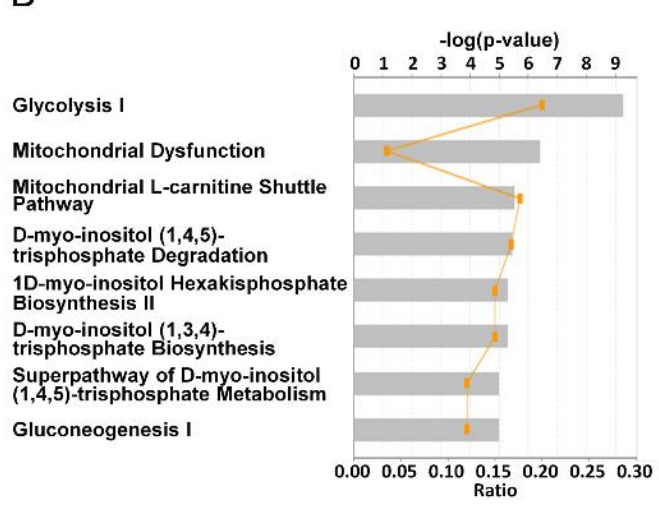

E

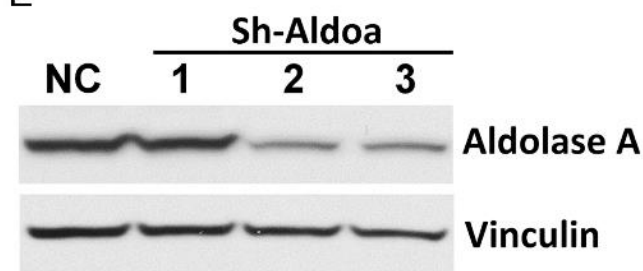

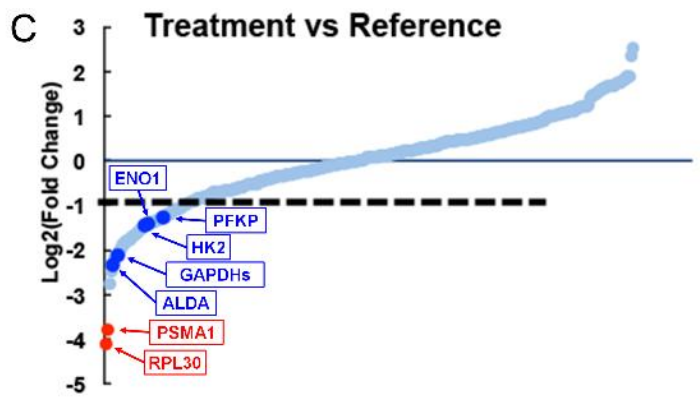

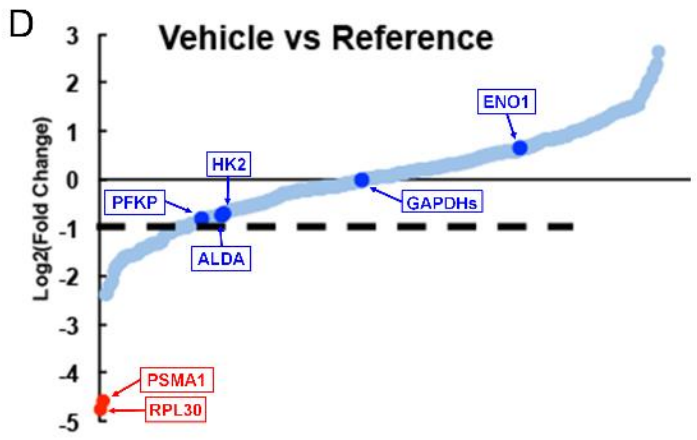

$\mathrm{F}$
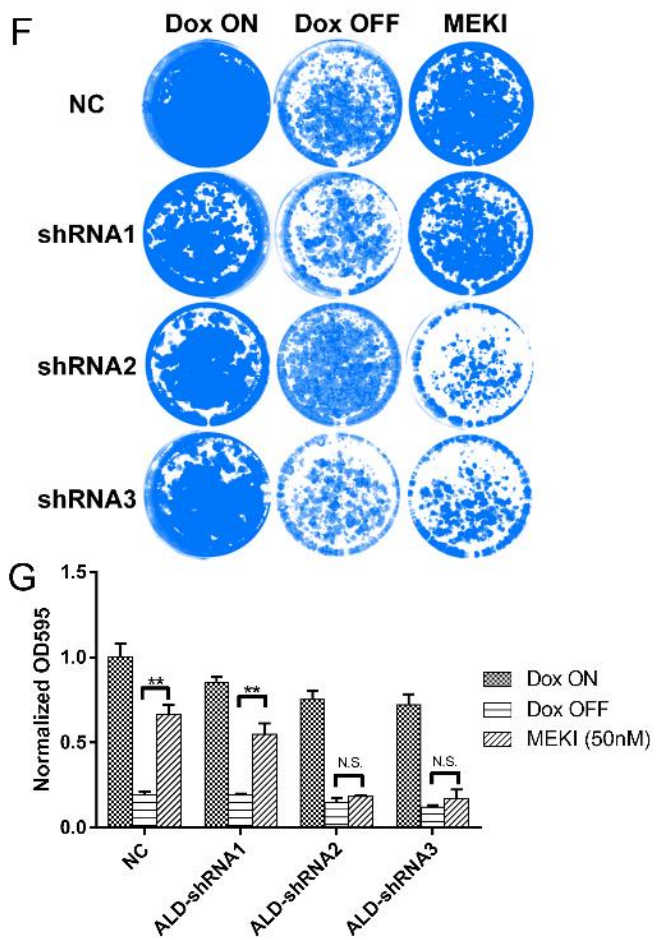

(A) Schematic illustration of pooled shRNA library screening in vivo. (B) IPA analysis of the depleted genes in shRNA library screen. (C-D) Relative abundance of each gene in the library in vehicle- or trametinib-treated tumors. Red dots: two positive control RPL30 and PSMA1; Blue Dots: five genes involved in glycolysis flux. (E) Knockdown of AldoA in iKras cell by shRNA. (F) Crystal violet staining of AldoA knockdown cell upon treatment of Dox ON, Dox OFF or 25 nM TRA for 4 days. (G) Quantification of crystal violet staining in $F(n=3$, Mean $\pm S D)$.

637 

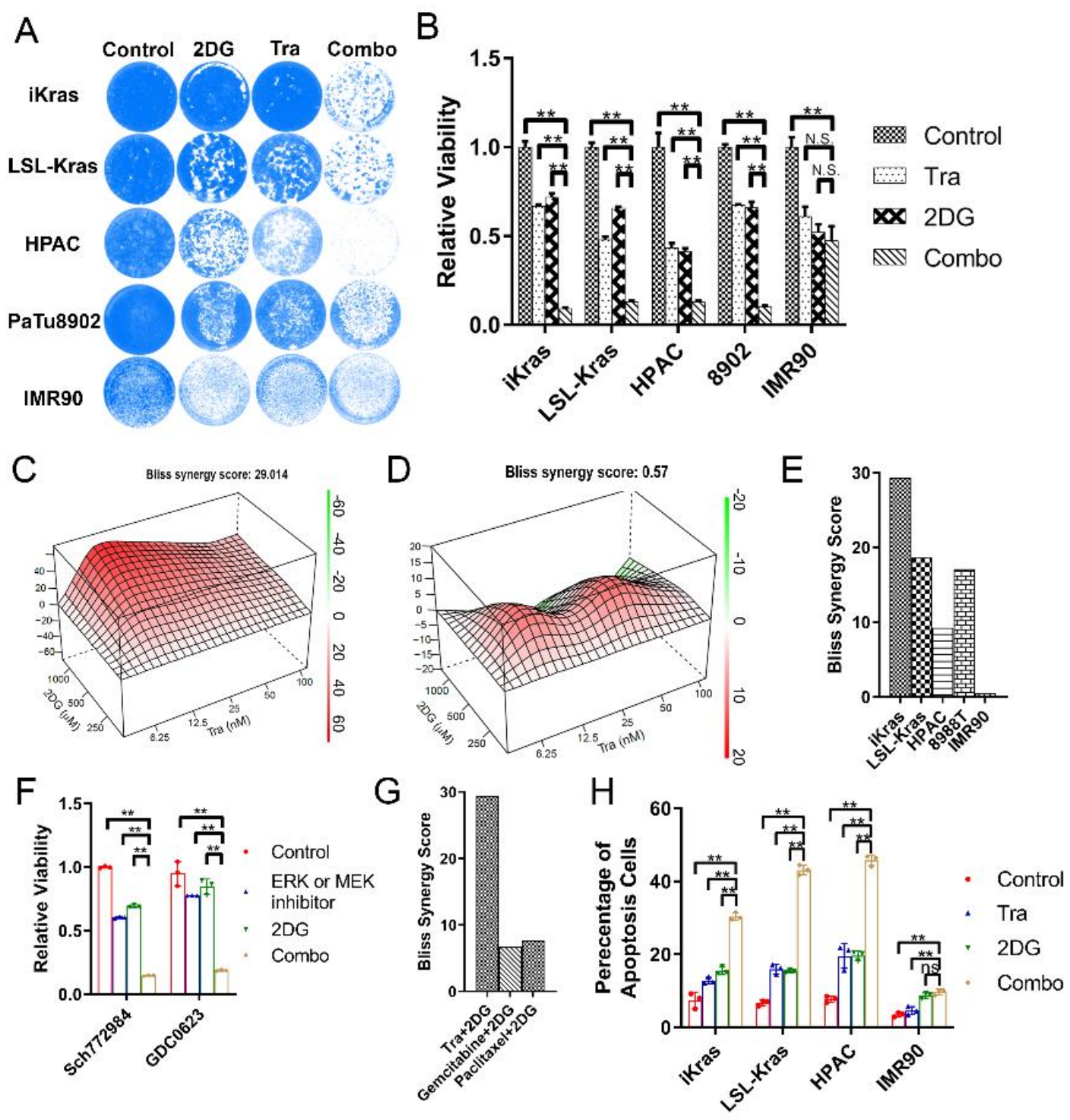

Figure 4. TRA and 2DG has a synergistic effect to induce apoptosis in mouse or human PDAC cell lines.

(A) Crystal violet staining of the cells treated with 25nM TRA, 1mM 2DG or combination for 4 days. (B) Quantification of crystal violet staining in B ( $n=3$, Mean \pm SD). (C-D) Representative Bliss score for combination of TRA and 2DG in iKras or IMR90 cell. (E) The synergistic effect of TRA and 2DG combination was analyzed using Bliss score in mouse (iKras and LSL-Kras), human PDAC line (HPAC and 8988T) and IMR90 cell line. (F) Quantification of crystal violet staining for the cell treated with SCH772984 (200nM)/GDC063 (100nM), 2DG (1mM) or combination (n=3, Mean \pm SD). (G) The

647 Bliss synergy score was calculated for the combination of TRA/2DG, gemcitabine/2DG and 648 paclitaxel/2DG in iKras cell. $(\mathrm{H})$ Apoptosis of the cell treated with $25 \mathrm{nM}$ TRA, 1mM 2DG or 649 combination by annexin V/7-AAD staining $(n=3$, Mean $\pm S D)$. 

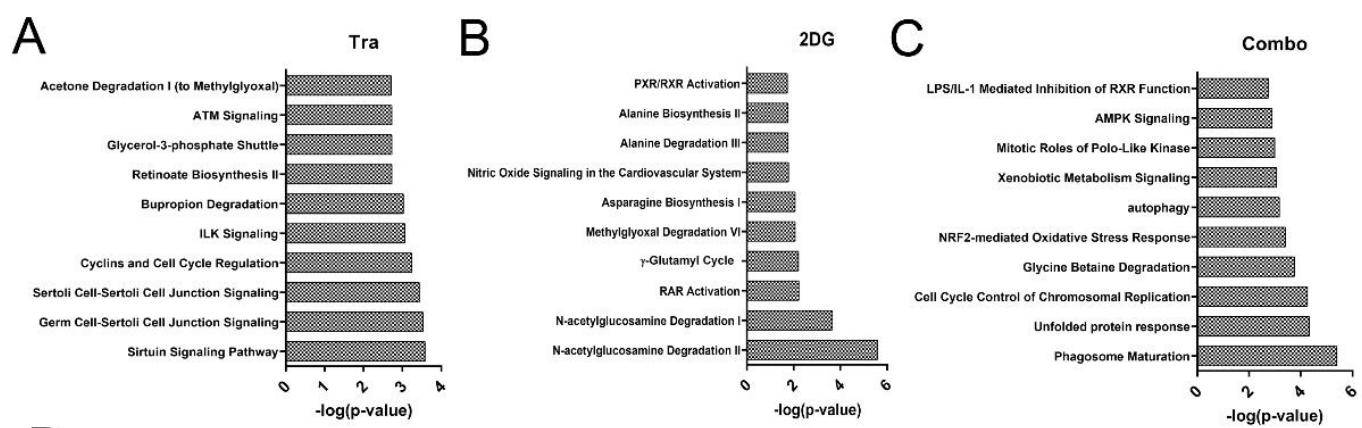

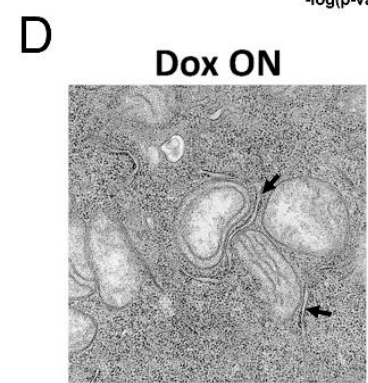

$E$
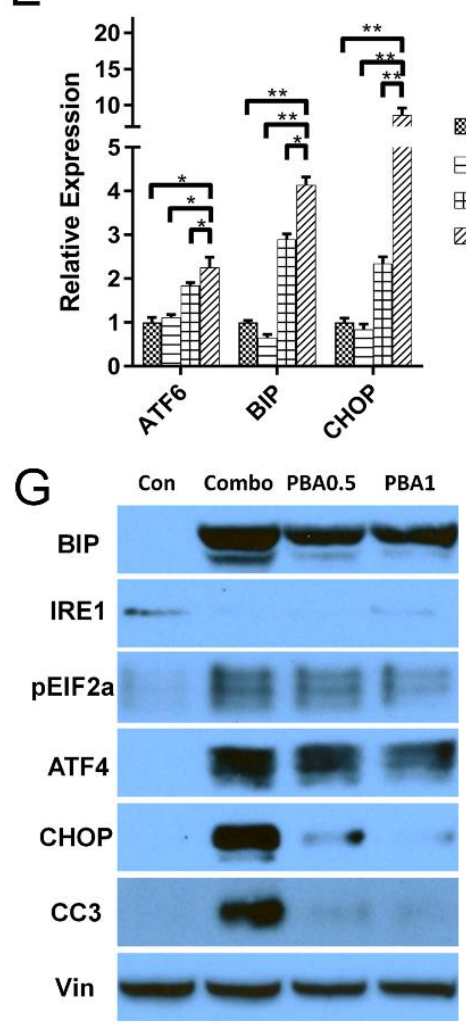

Tra

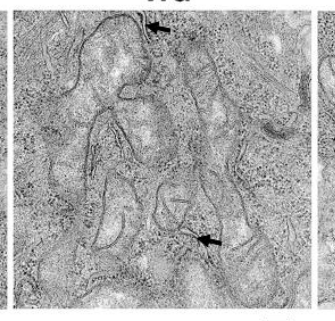

$\mathrm{F}$

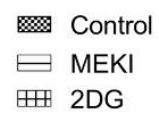

एII Combo
652

653

654

655

656

657

658

659

660

661

Figure 5. 2DG and TRA has a synergy to induce ER stress in PDAC cell lines.

(A-C) IPA analysis of differentially expressed genes in iKras lines treated with 2DG and TRA combination. (D) Representative TEM images of iKras cell treated with TRA, 2DG or Combination for 48h. The normal ER were indicated by arrow and swollen ER were indicates by arrowheads. (E-F) Expression of the ER stress markers in iKras treated with 25nM TRA, 1mM 2DG or combination for $48 \mathrm{~h}$ detected by $q P C R(E, n=3$, Mean \pm SD) or WB $(F)$. (G) Expression of the ER stress markers in iKras treated with TRA/2DG or TRA/2DG/PBA by WB. (H) Apoptosis analysis of iKras cell treated with TRA/2DG or TRA/2DG/PBA by Annexin-V staining ( $\mathrm{n}=3$, Mean \pm SD). TRA: $25 \mathrm{nM}$; 2DG: $1 \mathrm{mM}$ and PBA: $0.5 \mathrm{mM}$ or $1 \mathrm{mM}$. 


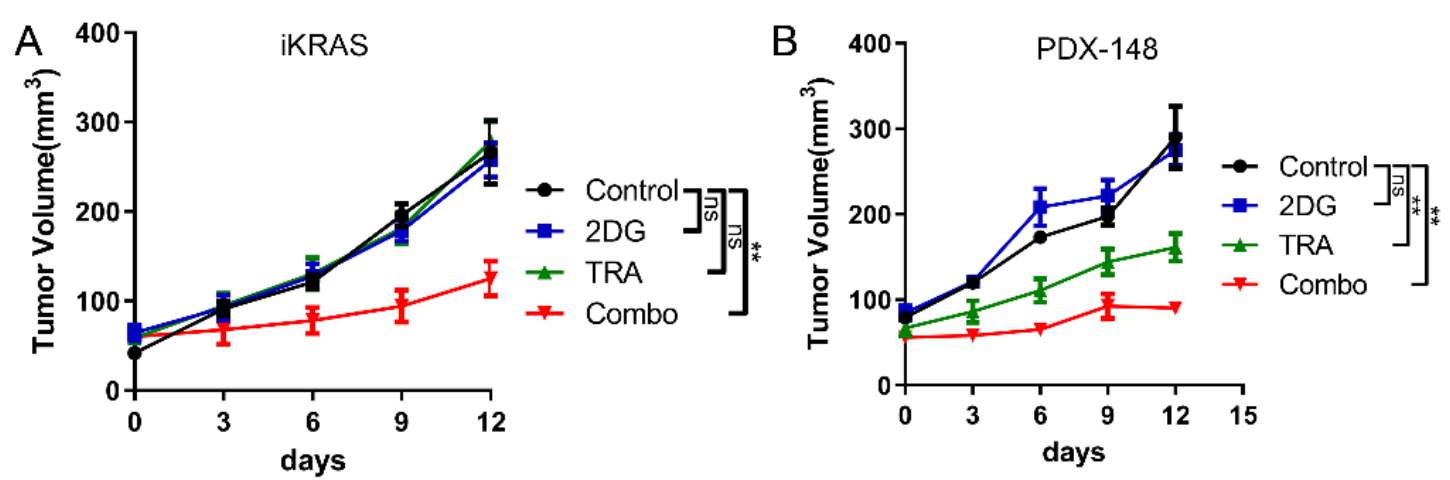

C
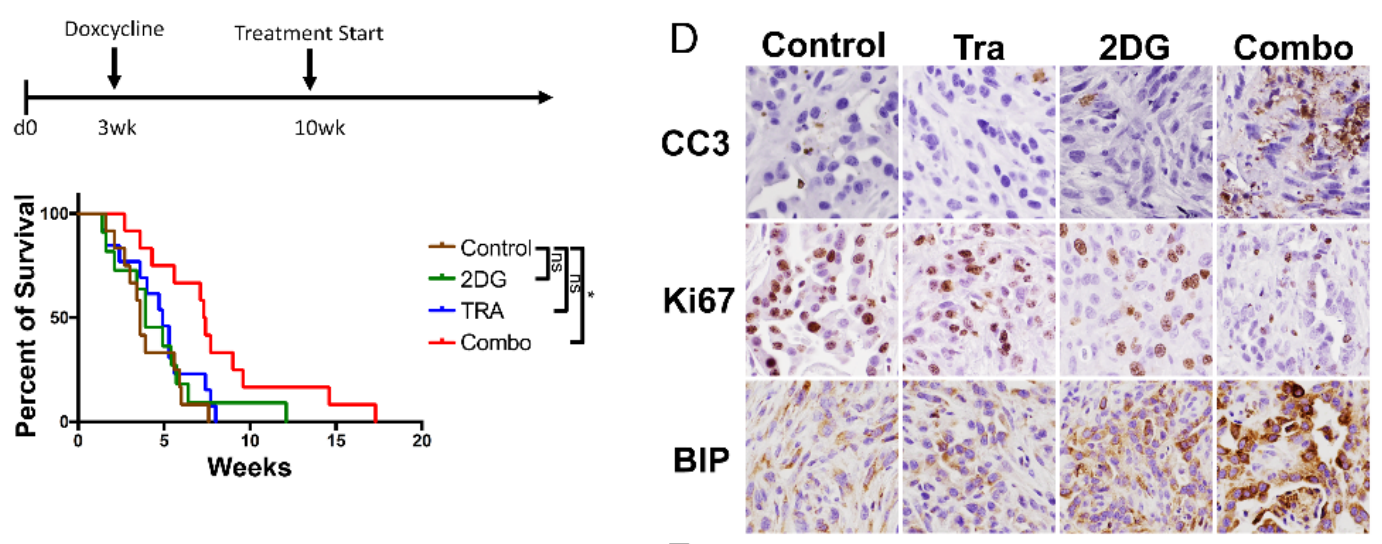

E

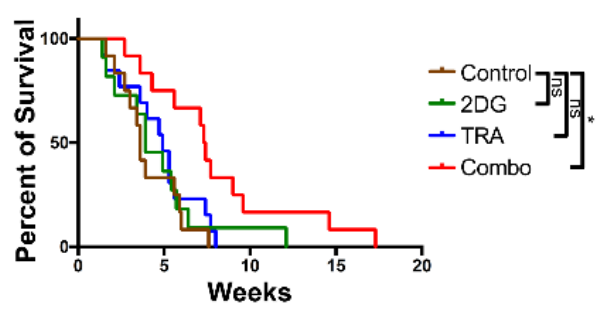

$\mathrm{F}$
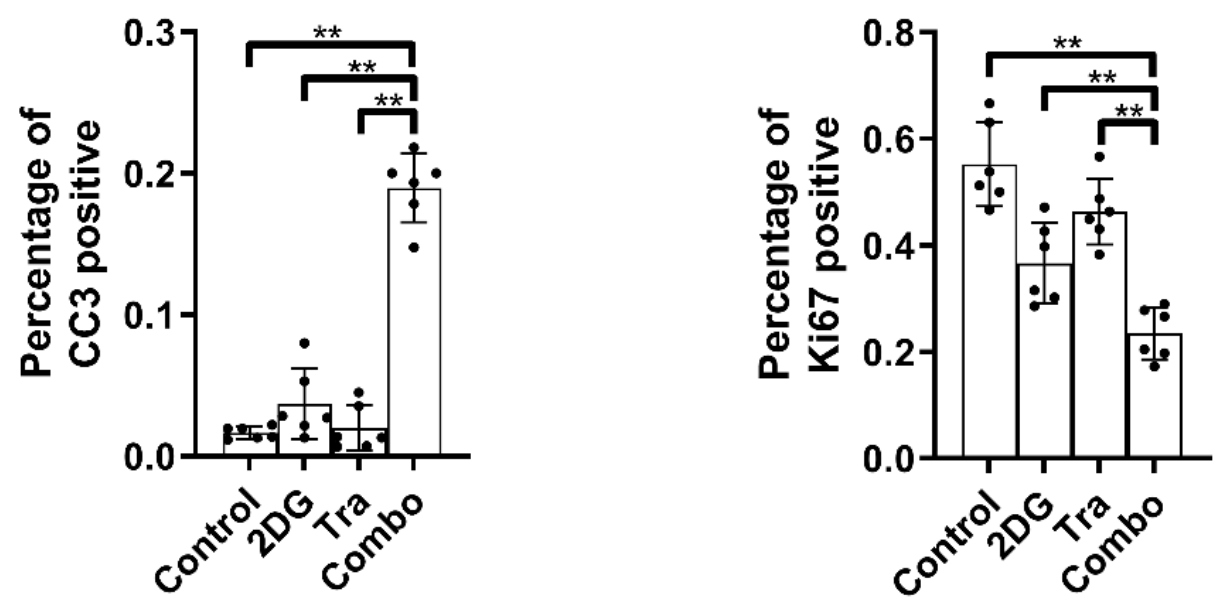

662

663

Figure 6. 2DG and MEK inhibitor combination has a synergy to treat PDAC in vivo.

664 (A) Xenograft tumor volume of iKras cell in nude mice treated with TRA, 2DG or Combo ( $\mathrm{n}=5$, Mean

$665 \pm$ SE). TRA: $1 \mathrm{mg} / \mathrm{kg} / \mathrm{day} ; 2 \mathrm{DG}: 1000 \mathrm{mg} / \mathrm{kg} / \mathrm{d}$. (B) Xenograft tumor volume of PDX-148 cell in nude 666 mice treated with TRA, 2DG or Combo ( $\mathrm{n}=5$, Mean \pm SE). TRA: $1 \mathrm{mg} / \mathrm{kg} / \mathrm{day} ; 2 \mathrm{DG}: 1000 \mathrm{mg} / \mathrm{kg} / \mathrm{d}$. (C) 667 Kaplan-Meier survival analysis of iKras GEMM treated with vehicle, TRA, 2DG or Combo (n=12). 668 TRA: $1 \mathrm{mg} / \mathrm{kg} / \mathrm{day} ; 2 \mathrm{DG}$ : 1000mg/kg/d. (D) Representative images of immunohistochemical staining 669 of paraffin embedded xenograft tumors using antibodies for CC3, Ki67 or BIP. (E-F) Quantification of 670 CC3 or ki67 positive cell in $\mathrm{D}(\mathrm{n}=6$, Mean \pm SD). 